\title{
Front Matter: Volume 9727
}

, "Front Matter: Volume 9727," Proc. SPIE 9727, Laser Resonators, Microresonators, and Beam Control XVIII, 972701 (1 July 2016); doi: $10.1117 / 12.2239514$

SPIE. Event: SPIE LASE, 2016, San Francisco, California, United States 


\title{
PROCEEDINGS OF SPIE
}

\section{Laser Resonators, Microresonators, and Beam Control XVIII}

\author{
Alexis V. Kudryashov \\ Alan H. Paxton \\ Vladimir S. Ilchenko \\ Lutz Aschke \\ Editors
}

\section{5-18 February 2016}

San Francisco, California, United States

Sponsored and Published by

SPIE 
The papers in this volume were part of the technical conference cited on the cover and title page. Papers were selected and subject to review by the editors and conference program committee. Some conference presentations may not be available for publication. Additional papers and presentation recordings may be available online in the SPIE Digital Library at SPIEDigitallibrary.org.

The papers reflect the work and thoughts of the authors and are published herein as submitted. The publisher is not responsible for the validity of the information or for any outcomes resulting from reliance thereon.

Please use the following format to cite material from these proceedings:

Author(s), "Title of Paper," in Laser Resonators, Microresonators, and Beam Control XVIII, edited by Alexis V. Kudryashov, Alan H. Paxton, Vladimir S. Ilchenko, Lutz Aschke, Proceedings of SPIE Vol. 9727 (SPIE, Bellingham, WA, 2016) Six-digit Article CID Number.

ISSN: 0277-786X

ISSN: 2410-9045 (electronic)

ISBN: 9781628419627

Published by

SPIE

P.O. Box 10, Bellingham, Washington 98227-0010 USA

Telephone +1 3606763290 (Pacific Time) · Fax +1 3606471445

SPIE.org

Copyright (C) 2016, Society of Photo-Optical Instrumentation Engineers.

Copying of material in this book for internal or personal use, or for the internal or personal use of specific clients, beyond the fair use provisions granted by the U.S. Copyright Law is authorized by SPIE subject to payment of copying fees. The Transactional Reporting Service base fee for this volume is $\$ 18.00$ per article (or portion thereof), which should be paid directly to the Copyright Clearance Center (CCC), 222 Rosewood Drive, Danvers, MA 01923. Payment may also be made electronically through CCC Online at copyright.com. Other copying for republication, resale, advertising or promotion, or any form of systematic or multiple reproduction of any material in this book is prohibited except with permission in writing from the publisher. The CCC fee code is 0277-786X/16/\$18.00.

Printed in the United States of America.

Publication of record for individual papers is online in the SPIE Digital Library.

\section{SPIE. DIGITAL \\ SPIEDigitalLibrary.org}

Paper Numbering: Proceedings of SPIE follow an e-First publication model. A unique citation identifier (CID) number is assigned to each article at the time of publication. Utilization of CIDs allows articles to be fully citable as soon as they are published online, and connects the same identifier to all online and print versions of the publication. SPIE uses a six-digit CID article numbering system structured as follows:

- The first four digits correspond to the SPIE volume number.

- The last two digits indicate publication order within the volume using a Base 36 numbering system employing both numerals and letters. These two-number sets start with 00, 01, 02, 03, 04, 05, 06, 07, 08, 09, OA, OB ... 0Z, followed by 10-1Z, 20-2Z, etc. The CID Number appears on each page of the manuscript. 


\title{
Contents
}

\author{
vii Authors \\ ix Conference Committee
}

\section{SESSION 1 NOVEL MICRORESONATOR OPTICS I}

972703 Long period gratings based frequency selective interrogation of micro-resonators along the same fiber [9727-2]

972704 Development of packaged silica microspheres coupled with tapered optical microfibers [9727-3]

972705 Observation of optically induced transparency in a micro-cavity (Invited Paper) [9727-4]

972706 Deterministic photon-atom and photon-photon interactions based on single-photon Raman interaction (Invited Paper) [9727-5]

\section{SESSION 2 NOVEL MICRORESONATOR OPTICS II}

972707 High-Q GRIN resonators (Invited Paper) [9727-6]

9727 OA III-V-semiconductor vertically-coupled whispering-gallery mode resonators made by selective lateral oxidation [9727-9]

SESSION 3 MICRORESONATOR FREQUENCY COMBS I: JOINT SESSION WITH CONFERENCES 9727 AND 9731

9727 OC Harmonic mode locking in a high-Q whispering gallery mode microcavity (Invited Paper) [9727-11]

SESSION 4 MICRORESONATOR FREQUENCY COMBS II: JOINT SESSION WITH CONFERENCES 9727 AND 9731

$9727 \mathrm{OH} \quad$ Optical frequency comb and spectroscopy with crystalline resonators in MIR (Invited Paper) [9727-16]

\section{SESSION 5 FREQUENCY COMBS AND LASERS}

9727 OK Third order nonlinear phenomena in silica solid and hollow whispering gallery mode resonators [9727-19] 
$9727 \mathrm{OL} \quad$ Multi-scale nonlinear effects in whispering-gallery mode resonators [9727-20]

9727 ON Novel ultrafast sources on chip: filter driven four wave mixing lasers, from high repetition rate to burst mode operation (Invited Paper) [9727-22]

\section{SESSION 6 OPTOMECHANICS WITH MICRORESONATORS}

$972700 \quad$ Using mechanics to convert between microwave and optical frequencies (Invited Paper) [9727-23]

\section{SESSION $7 \quad$ BEAM SHAPING I: JOINT SESSION WITH CONFERENCES 9727 AND 9741}

9727 OS Field mappers for laser material processing [9727-27]

9727 OT Specialty flat-top beam delivery fibers with controlled beam parameter product [9727-28]

9727 OU Real time m-square and beam parameter product measurement using GigE CMOS sensors [9727-29]

\section{SESSION $8 \quad$ BEAM SHAPING II: JOINT SESSION WITH CONFERENCES 9727 AND 9741}

9727 OW Flexible assembly module for beam-shaping product families based on support structures [9727-31]

\section{SESSION 9 MICRORESONATORS: QUANTUM AND NONLINEAR PHENOMENA AND APPLICATIONS}

972710 Efficient second harmonic generation in an on-chip high-Q crystalline microresonator fabricated by femtosecond laser [9727-35]

\section{SESSION 10 MICRORESONATOR SENSORS I}

972713 Whispering-gallery mode resonator sensors based on liquid droplets [9727-67]

\section{SESSION 11 MICRORESONATOR SENSORS II}

972716 Quantum dot optofluidic lasers and their prospects for biochemical sensing (Invited Paper) [9727-41]

972718 Flow sensing using a hollow whispering gallery mode microlaser [9727-43]

972719 Localized biomolecules immobilization in optical microbubble resonators [9727-44]

9727 1A Optical heterodyne detection for ultra-high Q micro-disk laser sensor [9727-45]

iv 
9727 1C Spinning optical resonator sensor for torsional vibrational applications measurements [9727-47]

SESSION 12 MODE CONTROL AND SELECTION AND BEAM CHARACTERIZATION AND CONTROL I

$9727 \mathrm{lH} \quad$ Beam control through nonlinear propagation in air and laser induced discharges (Invited Paper) [9727-51]

SESSION 13 MODE CONTROL AND SELECTION AND BEAM CHARACTERIZATION AND CONTROL II

$972711 \quad$ Largest in the world bimorph deformable mirror for high-power laser beam correction (Invited Paper) [9727-52]

9727 iJ Discrete excitation of mode pulses using a diode-pumped solid-state digital [9727-53]

9727 1K Intracavity generation of low-loss radial-order Laguerre-Gaussian modes using digital holograms [9727-54]

\section{POSTER SESSION}

$9727 \mathrm{IL}$ Thermal lensing measurement from the coefficient of defocus aberration using ShackHartmann wavefront sensor [9727-55]

9727 IN Investigations of a dual seeded 1178nm Raman laser system [9727-57]

972710 The finite-difference matrix for beam propagation: eigenvalues and eigenvectors [9727-58]

9727 is Influence of the sensitivity of an optical resonator with a surface layer by its properties [9727-62]

9727 IT Time-domain observation of strong coupling between counter-propagating ultra-high Q whispering gallery modes [9727-63]

9727 IV Problems of uniform focal spot formation by means of deformable mirror [9727-65] 
Proc. of SPIE Vol. $9727972701-6$

Downloaded From: https://www.spiedigitallibrary.org/conference-proceedings-of-spie on 26 Apr 2023 Terms of Use: https://www.spiedigitallibrary.org/terms-of-use 


\section{Authors}

Numbers in the index correspond to the last two digits of the six-digit citation identifier (CID) article numbering system used in Proceedings of SPIE. The first four digits reflect the volume number. Base 36 numbering is employed for the last two digits and indicates the order of articles within the volume. Numbers start with 00, 01, 02, 03, 04, 05, 06, 07, 08, 09, 0A, OB...0Z, followed by 10-1Z, 20-2Z, etc.

Aas, Mehdi, 16

Abramczyk, J., OT

Äit-Ameur, Kamel, 1K

Aleksandrov, Alex, 11

Ali, Amir R., 1C

Almuneau, G., OA

Arissian, Ladan, $1 \mathrm{H}$

Arlotti, C., OA

Armani, A. M., 07

Arnoult, A., OA

Avino, S., 13

Baker, Howard J., OS

Baldini, F., 03, 19

Barucci, A., OK, 19

Bechler, Orel, 06

Becker, F., OT

Beleke, Andreas, OW

Belke, S., OT

Bell, Teboho, 1J, 1K, $1 \mathrm{~L}$

Berneschi, S., OK, 19

Blair, Paul, OS

Block, Matthew, $1 \mathrm{~N}$

Borsoni, Giles, 11

Brambilla, Gilberto, 04

Brecher, Christian, OW

Calmon, P.-F., OA

Calvez, S., OA

Cao, Jianjun, 05

Chembo, Yanne K., OL

Chen, Qiushu, 16

Cheng, Ya, 10

Chen-Jinnai, Akitoshi, OC, $1 \mathrm{~T}$

Chiavaioli, F., 03

Choi, H., 07

Choi, Young-Wan, 1A

Chu, Sai T., ON

Cleland, A. N., 00

Conroy, M., OT

Cosci, A., 19

Cosi, F., 03, OK, 19

Currie, Matthew, OS

Dayan, Barak, 06

De Natale, P., 13

Diallo, Souleymane, $\mathrm{OL}$

Diels, Jean-Claude, $1 \mathrm{H}$

Diep, V., 07

Fan, Xudong, 16

Fang, Wei, 10

Fang, Zhiwei, 10
Farley, K., ОT

Farnesi, D., 03, OK, 19

Farrell, Gerald, 04

Feng, Chengyong, $1 \mathrm{H}$

Forbes, Andrew, 1K, $1 \mathrm{~L}$

Fujii, Shun, IT

Gagliardi, G., 13

Gatherer, Andrew, 1C

Gauthier-Lafaye, O., OA

Giannetti, A., 19

Giorgini, A., 13

Grudinin, Ivan S., $\mathrm{OH}$

Guendelman, Gabriel, 06

Haag, Sebastian, OW

Haas, Gil, OU

Haverkamp, Tobias, OW

Henry, Leanne J., 1N

Ibrahim, Mariam S., 1C

Jain, Ravinder, $1 \mathrm{~N}$

Ji, Myung-Gi, 1 A

Jitsuno, Takahisa, 11

Jollivet, C., OT

Jonáš, Alexandr, 16

Jung, Mi, 1A

Kamer, Brian, $1 \mathrm{H}$

Kasparian, Jerome, $1 \mathrm{H}$

Kato, Takumi, OC

Kim, Chil-Min, 1A

Kim, Hong-Seung, $1 \mathrm{~A}$

Kim, Tae-Ryong, 1 A

Kiraz, Alper, 16

Klopfer, Michael, iN

Kobatake, Tomoya, OC

Kovach, A., 07

Kudryashov, Alexis, 11, 1V

Kuo, K., 07

Lafleur, G., OA

Larrue, A., OA

Lin, Guoping, OL

Lin, Jintian, 10

Lovsky, Yulia, 06

Lylova, Anna, $1 \mathrm{~V}$

Madugani, Ramgopal, 04

Malara, P., 13

Mansour, Kamjou, $\mathrm{OH}$

McBride, Roy, OS

Mongin, Denis, $1 \mathrm{H}$

Morandotti, Roberto, ON

Moss, Dave J., ON 
Müller, Tobias, OW

Murphy, Eoin, OS

Naidoo, Darryl, $1 \mathrm{~L}$

Ngcobo, Sandile, 1J, 1K, $1 \mathrm{~L}$

Nic Chormaic, Síle, 04, 18

Nunzi Conti, G., 03, OK, 19

Oh, Kwang Ryong, 1A

Ostendorf, Andreas, is

Pasquazi, Alessia, ON

Paxton, Alan H., 10

Peairs, G. A., $0 \mathrm{O}$

Peccianti, Marco, ON

Pelli, S., 19

Rastegari, Ali, $1 \mathrm{H}$

Righini, G. C., 03, OK, 19

Romanov, Pavel, 11

Rosenblum, Serge, 06

Rübenach, Olaf, OW

Rukosuev, Alexey, IV

Samarkin, Vadim, 1I, IV

Satzinger, K. J., 00

Scaggs, Michael, OU

Schubert, Elise, $1 \mathrm{H}$

Schweiger, Gustav, is

Sheldakova, Julia, 1I, IV

Shomroni, Itay, 06

Soltani, S., 07

Son, Byung-Hee, $1 \mathrm{~A}$

Soria, Silvia, 03, 0K, 19

Suzuki, Ryo, OC

Tanabe, Takasumi, OC, IT

Tankala, K., OT

Tetsumoto, Tomohiro, $1 T$

Tombelli, S., 19

Trela, Natalia, OS

Trono, C., 03, 19

Vainsencher, A., 00

Walker, Duncan, OS

Wan, Wenjie, 05

Wang, Min, 10

Wang, Pengfei, 04

Ward, Jonathan M., 04, 18

Weigel, Thomas, is

Wenzel, Christian, OW

Wolf, Jean-Pierre, $1 \mathrm{H}$

$\mathrm{XU}$, Yingxin, 10

Yang, Yong, 04, 18

Yoshiki, Wataru, $1 T$

$\mathrm{Yu}, \mathrm{Nan}, \mathrm{OH}$

Zhao, Haoyu, 04

Zheng, Yuanlin, 05

Zontar, Daniel, OW

Zullo, R., 13 


\section{Conference Committee}

Symposium Chairs

Guido Hennig, Daetwyler Graphics AG (Switzerland)

Yongfeng Lu, University of Nebraska-Lincoln (United States)

Symposium Co-chairs

Reinhart Poprawe, Fraunhofer-Institut für Lasertechnik (Germany)

Koji Sugioka, RIKEN (Japan)

Program Track Chair

Bo Gu, Bos Photonics (United States)

Conference Chairs

Alexis V. Kudryashov, Moscow State Open University

(Russian Federation)

Alan H. Paxton, Air Force Research Laboratory (United States)

Vladimir S. Ilchenko, OEwaves, Inc. (United States)

Conference Co-chair

Lutz Aschke, IVAM Microtechnology Network (Germany)

Conference Program Committee

Andrea M. Armani, The University of Southern California (United States)

Gaurav Bahl, University of Illinois at Urbana-Champaign (United States)

Yanne K. Chembo, FEMTO-ST (France)

Jean-Claude M. Diels, The University of New Mexico (United States)

Hans Joachim Eichler, Laser- und Medizin-Technologie GmbH, Berlin (Germany)

Andrew Forbes, CSIR National Laser Center (South Africa)

Pierre Galarneau, INO (Canada)

Thomas Graf, University Stuttgart (Germany)

Tobias J. Kippenberg, Ecole Polytechnique Fédérale de Lausanne (Switzerland)

James R. Leger, University of Minnesota, Twin Cities (United States)

Andrey B. Matsko, OEwaves, Inc. (United States)

Gualtiero Nunzi Conti, Istituto di Fisica Applicata Nello Carrara (Italy) 
Andrew W. Poon, Hong Kong University of Science and Technology (Hong Kong, China)

Michelle L. Povinelli, The University of Southern California

(United States)

Michael J. Scaggs, Neoteric Concepts, LLC (United States)

Haiyin Sun, Chemlmage Corporation (United States)

Kunihiko Washio, Paradigm Laser Research Ltd. (Japan)

Yun-Feng Xiao, Peking University (China)

Lei Xu, Fudan University (China)

Lan Yang, Washington University in St. Louis (United States)

\section{Session Chairs}

1 Novel Microresonator Optics I

Vladimir S. Ilchenko, OEwaves, Inc. (United States)

2 Novel Microresonator Optics II

Andrey B. Matsko, OEwaves, Inc. (United States)

3 Microresonator Frequency Combs I: Joint Session with Conferences 9727 and 9731

Andrea M. Armani, The University of Southern California (United States)

4 Microresonator Frequency Combs II: Joint Session with Conferences 9727 and 9731

Konstantin L. Vodopyanov, CREOL, The College of Optics and Photonics, University of Central Florida (United States)

5 Frequency Combs and Lasers

Tobias J. Kippenberg, Ecole Polytechnique Fédérale de Lausanne (Switzerland)

6 Optomechanics with Microresonators

Andrey B. Matsko, OEwaves, Inc. (United States)

7 Beam Shaping I: Joint Session with Conferences 9727 and 9741

Lutz Aschke, IVAM Microtechnology Network (Germany)

8 Beam Shaping II: Joint Session with Conferences 9727 and 9741

Lutz Aschke, IVAM Microtechnology Network (Germany)

9 Microresonators: Quantum and Nonlinear Phenomena and Applications

Lutz Aschke, IVAM Microtechnology Network (Germany) 
10 Microresonator Sensors I

Vladimir S. Ilchenko, OEwaves, Inc. (United States)

11 Microresonator Sensors II

Andrew W. Poon, Hong Kong University of Science and Technology (Hong Kong, China)

12 Mode Control and Selection and Beam Characterization and Control I

Alexis V. Kudryashov, Moscow State Open University (Russian Federation)

13 Mode Control and Selection and Beam Characterization and Control II

Alan H. Paxton, Air Force Research Laboratory (United States) 
Proc. of SPIE Vol. $9727972701-12$

Downloaded From: https://www.spiedigitallibrary.org/conference-proceedings-of-spie on 26 Apr 2023 Terms of Use: https://www.spiedigitallibrary.org/terms-of-use 